\title{
Status of Serum Leptin and Lipid Profile in Newly Diagnosed Psoriasis Patients: A Case Control Study \\ (Original Research Paper)
}

\author{
Authors \\ Ghalaut VS ${ }^{1}$, Sarkar $M^{2}$, Dayal $S^{3}$, Gupta $G^{4}$, Samanta $S^{5}$, Rajju $T^{6}$ \\ ${ }^{1}$ Professor and Head, Department of Biochemistry, PT. B. D. SHARMA PGIMS Rohtak, INDIA \\ Email: veenaghalaut@yahoo.co.in \\ ${ }^{2}$ JR,Department of Biochemistry, PT. B. D. SHARMA PGIMS Rohtak, INDIA \\ Email: madhumitaoct16@gmail.com \\ ${ }^{3}$ Associate Professor, Department of Dermatology, PT. B. D. SHARMA PGIMS Rohtak, INDIA \\ Email: sd_md_mini@yahoo.com \\ ${ }^{4}$ Senior resident, Department of Biochemistry, AIIMS, Jodhpur, INDIA \\ Email:dr.garnick@gmail.com \\ ${ }^{5}$ Senior Resident, Department of Critical Care Medicine SGPGIMS,Lucknow, INDIA \\ Email:dr.sujay14@gmail.com \\ ${ }^{6}$ JR.Department of Biochemistry, PT. B. D. SHARMA PGIMS Rohtak, INDIA \\ Email:rajju.tiwari55@gmail.com \\ Corresponding Author \\ MadhumitaSarkar \\ Junior Resident, Department of Biochemistry, PT. B. D. SHARMA PGIMS Rohtak, INDIA \\ Address: 17, Dr. A N Paul Lane, Bally, Howrah,West Bengal- 711201 \\ Email:madhumitaoct16@gmail.com, Mobile no.918685994283
}

\begin{abstract}
Objective: Psoriasis is an autoimmune chronic inflammatory disease involving the skin and mucous membranes. Besides the autoimmune and genetic factors many stressful physiologic, psychological events and environmental factors play an important role in disease etiopathogenesis. Leptin, an adipocyte-derived hormone, plays a role in immune responses and may be involved in the pathogenesis of psoriasis. Psoriasis is associated with variety of lipid profile disorders. So, weperformed this study to determine serum leptin levels and lipid profile in psoriasis patients.

Material and methods: We conducted a prospective case control study from February 2014 to April 2015 on 30 patients of psoriasis and 30 age and sex match healthy control subjects. We measured serum leptin level by ELISA technique and lipid profile by enzymatic method.

Results: Serum leptin and lipid profile of total 60 subjects, 30 patients of psorisis and 30 age and sex match healthy volunteers were analysed in this study. Leptin level was found to be significantly higher in psorisis group as compared to healthy volunteers $(p<0.05)$. We also found significantly higher leptin level in psoriasis patients with higher PASI(Psoriasis Area and Severity Index) score (>15) compared to low PASI score (< 15).Among lipid profile parameters TC, TG, LDL-C and VLDL-C were significantly higher in psoriasis group compared to healthy control ( $p<0.05)$. HDL-C level was significantly lower in psoriasis patients compared to
\end{abstract}


healthy controls $(p<0.05)$.

Conclusion:Psoriasis is associated with hyperleptinemia. Higher leptin level was found in patients with higher PASI score. Thus, it may have role in the pathogenesis or severity of psoriasis. Dyslipidemia is also common in psoriasis. We recommend early monitoring of both leptin and lipid profile in these patients to prevent development of metabolic syndrome and long term cardiovascular events. We suggest more large scale studies in psoriasis patients to establish it.

Keywords: Leptin, Lipid profile, Psoriasis.

\section{Introduction}

Psoriasis is a common, chronic, disfiguring, inflammatory and proliferative condition of the skin, in which both genetic and environmental factors play a critical role. ${ }^{[1]}$ It exists as several morphological variants like plaque, pustular, guttate and flexural. Some other variety also exist like nail psoriasis, drug induced, inverse psoriasis, psoriatic arthritis and seborrheic.Approximately 90 percent of affected patients have plaque psoriasis, characterized by well-defined round or oval plaques that differ in size and often coalesce. ${ }^{[2]}$ Males and females are equally affected by psoriasis vulgaris. Smoking increases the risk of psoriasis and its severity. ${ }^{[3,}{ }^{4]} \mathrm{BMI}$ is an important associated factor and a positive association between BMI and psoriasis has been reported. ${ }^{[5]}$ The Psoriasis Area and Severity Index (PASI) is a most widely used quantitative rating score for measuring the severity of psoriatic lesions based on area coverage and plaque appearance.

Leptin a polypeptide mainly produced and secreted by white adipose tissue in proportion to body fat mass. ${ }^{[6]}$ Higher leptin levels in psoriatic patients may be related to the fact that leptin and its receptors have structural similarity with cytokine type -1 superfamily and plays role in immune response and T-lymphocytes homing in lesional dermis and epidermis. ${ }^{[1,7-8]}$ Leptin, through modulation of synthesis of cytokines and inhibiting synthesis of Th 2(Helper T Lymphocyte 2 ), cells, might be involved in the pathogenesis of psoriasis. ${ }^{[9,10]}$

Cerman et al reported that serum leptin, tissue leptin and leptin receptor expression in psoriasis is a marker of severity and chronicity and its antagonist may play a role in adjuvant treatment in psoriasis with obesity. ${ }^{[11]}$ Zayed et al also reported that there is a strong positive correlation between the leptin levels and tumour necrosis factor- $\alpha$ (TNF- $\alpha$ ) and interleukin (IL)-6 in psoriasis vulgaris patients. ${ }^{[12]}$

Psoriasis is associated with cardio metabolic risk factors, such as dyslipidaemia, central obesity and insulin resistance. Psoriasis also increases the risk of cardiovascular disease (CVD). ${ }^{[13,14]}$ The association may be based on pathophysiology, shared risk factors or even treatment for psoriasis. Proinflammatory cytokines found to be increased in the blood and skin of patients with psoriasis including TNF- $\alpha$, IL-6 and IL-17 have been implicated in adipose tissue dysfunction and insulin resistance in central obesity. ${ }^{[15]}$ Commonly reported lipid abnormalities in patients with psoriasis include: elevated LDL-C, TC and triglyceride (TG) levels as well as decreased HDL-C levels. ${ }^{[16]}$

Thus in the present study we planned to analyze serum leptin levels and lipid profile in newly diagnosed psoriasis patients and to assess the association of leptin level with disease severity.

\section{Materials and Methods}

This prospective case control study was conducted in Rohtak, India from February 2014 to April 2015. Written informed consent was taken from all subjects included in the study.

We prospectively recruited new clinically diagnosed cases of psoriasis patients, not on any treatment for last four weeks as subjects after meeting the inclusion criteria during the study period. We selected age- and sex-matched healthy controls from healthy hospital staffs not affected by psoriasis or other autoimmune diseases. We 
included our study subjects aged between 20 to 60years.

We excluded all known cases of kidney disease, liver disease, ischemic heart disease, neurological disease, endocrine disorders, malignancy, Polycystic ovarian syndrome, pregnant or lactating women, patients undergone major surgery or trauma and subjects having drug addiction. Patients under treatment with systemic corticosteroids, lipid lowering agents, thiazides, retinoids, beta blockers, methotrexate, cyclosporine and immunosuppressive were also excluded from the study.

Serum leptin and lipid profile were done before the start of treatment in psoriasis patients. Complete history and physical examination with anthropometry were also performed in cases and controls. Clinical severity of disease was assessed using PASI scoring. Accordingly, mild-moderate psoriasis and severe psoriasis were defined as PASI $<15$ and PASI $\geq 15$, respectively.

Data collection: Detailed history regarding all demographic parameters (age, sex, BMI, socioeconomic status, addictions, duration of disease), any systemic illness or comorbidities, any drug intake were taken. Clinical severity of disease was assessed using Psoriasis Area and Severity Index (PASI) score. Serum leptin, lipid profile and routine biochemical parameters were measured.We recorded all relevant data.

\section{Sample Collection and Storage}

Six $\mathrm{mL}$ of fasting (12 hr fasting) venous blood sample was taken in a plain red capped evacuated blood collection tube under all aseptic precautions. Samples were processed within one hour of collection. Serum was separated by centrifugation at $2000 \mathrm{rpm}$ for 10 minutes after clotting. Routine investigations were done on the same day and rest of the serum stored at $-200 \mathrm{C}$ for serum leptin estimation for subsequent analysis. All routine investigations including lipid profile were done in autoanalyzer using enzymatic method.

\section{Serum Leptin}

DRG Leptin ELISA (enzyme immunoassay for quantitative in vitro diagnostic measurement of Leptin in serum and plasma) kit was used. ${ }^{[17]}$

\section{Principle of the Test}

DRG Leptin ELISA kit is a solid phase enzyme linked immunosorbent assay based on the sandwich principle. Microtiter wells were coated with a monoclonal antibody directed towards a unique antigenic site on leptin molecule. An aliquot of patient sample containing endogenous leptin was incubated in specific well with biotinylated monoclonal anti Leptin antibody. A sandwich complex was formed after incubation the unbound material was washed off and a Peroxidise enzyme complex was added for detection of the bound leptin. After adding the substrate solution the intensity of color developed is proportional to the concentration of leptin in the sample.

\section{Serum Lipid Profile}

TG was estimated in autoanalyzer (Randox) by enzymatic method. The TG level determined after enzymatic hydrolysis with lipases. The indicator, quinoneimine formed from hydrogen-peroxide, 4aminophenazone and 4-chlorophenol under the catalytic influence of peroxidise. ${ }^{[18]}$ TC estimated by enzymatic method in autoanalyzer (Randox). The cholesterol is determined after enzymatic hydrolysis and oxidation. The indicator quinoneimine is formed from hydrogen peroxide and 4-aminoantipyrine in the presence of phenol and peroxidise. ${ }^{[18]}$ HDL estimated in autoanalyzer (Randox) by enzymatic method. The enzymes cholesterol esterase, cholesterol oxidase and peroxidase are used with 4-AA and HDAOS as indicator after chylomicron, VLDL-C and LDL-C is eliminated. ${ }^{[18]}$ LDL-C was determined by Friedewald equation. VLDL-C was obtained by formula. [19]

\section{Statistical Analysis}

Statistical analysis was done by standard statistical software SPSS version 18 using appropriate statistical tests. Data were expressed as Mean \pm $\mathrm{SD}$, frequency or number as appropriate. Pearson 
Chi Square Test was used for categorical variables. Comparison between two groups was analysed using student $t$ test or Mann Whitney $U$ test as appropriate. Pearson correlation was used to examine the strength of correlation among various risk factors, leptin level and lipid profile. Multiple logistic regression analysis was also applied where appropriate. For all statistical tests $\mathrm{P}$ value $<0.05$ was considered as significant.

\section{Results}

There were total 60 subjects, 30 subjects in each group comprising of patients with psoriasis and healthy volunteers respectively. Demographic parameters such as age, gender and BMI distribution were comparable between two groups (Table 1).Leptin level was significantly higher in psoriasis patients as compared to healthy volunteers $(20.02 \pm 11.69$ and $9.38 \pm 4.75$ respectively, $\mathrm{p}<0.05$; Table 2, Figure 1).

Among psoriasis patients females had slightly higher leptin levels as compared to males but statistically insignificant $(11.54 \pm 9.78$ vs $11.04 \pm$ 9.90; $p=0.814$, Table 3 ). In psoriasis group leptin level was significantly higher in patients with higher PASI score ( $>15)$ compared to low PASI score $(<15)$ [p<0.05, Table 4 and Figure 2] Serum leptin level was found to be higher in psoriasis patients with high BMI than low BMI group but statistically insignificant ( $p>0.05$, Table 5).
Among lipid profile parameters TC, TG, LDL-C and VLDL-C were significantly higher in psoriasis group compared to healthy control $(\mathrm{p}<0.05)$. HDL-C level was significantly lower in psoriasis patients compared to healthy controls ( $\mathrm{p}$ $<0.05$,Table 6). All lipid profile parameters were statistically comparable between normal and high BMI group in psoriatic patents. Similarly all lipid profile parameters were also statistically comparable between low and high PASI subgroup in psoriatic patents.

Leptin had a significant positive correlation with lipid profile parameters such as TG, VLDL-C ( $\mathrm{r}$ value for $\mathrm{TG}=0.398$, VLDL-C $=0.402$ ) $\mathrm{In}$ addition, leptin had also positive correlation with BMI $(r=0.128)$ and lipid profile parameters like $\mathrm{TC}$ and LDL-C ( $\mathrm{r}$ value for TC $=0.154, \mathrm{LDL}-\mathrm{C}=$ 0.150) while having significant negative correlation with HDL-C ( $\mathrm{r}$ value $=-0.423$ ) in our study population. (Table 7). BMI had nonsignificant positive correlation with most of the lipid profile parameters such as TC, TG, LDL$\mathrm{C}$ and VLDL-C except with the HDL-C having negative correlation (Table 8).

Multiple regression analysis was performed for leptin from independent variables like age, BMI, TC, TG, HDL-C and VLDL-C. Prediction of leptin from these variables was not statistically significant $[\mathrm{F}(6,23)=1.42, \mathrm{p}=0.248, \mathrm{R} 2=$ $0.271]$.

Table 1: Demographic characteristics

\begin{tabular}{|l|c|c|c|}
\hline Parameters & Psoriasis & Healthy volunteers & P value \\
\hline Gender(M/F)* & $18 / 12$ & $16 / 14$ & 0.602 \\
\hline Age (in year)\$ & $41.1 \pm 15.84$ & $39.9 \pm 7.92$ & 0.712 \\
\hline BMI $\left(\mathrm{kg} / \mathrm{m}^{2}\right) \$$ & $25.81 \pm 2.31$ & $25.09 \pm 2.07$ & 0.209 \\
\hline
\end{tabular}

*expressed in frequency, $\$$ expressed in Mean \pm SD

Table 2: Serum leptin level in two groups (Mean \pm SD)

\begin{tabular}{|l|l|l|l|}
\hline Parameters & Psoriasis $\quad(\mathrm{n}=30)$ & Healthy volunteers $\quad(\mathrm{n}=30)$ & P value \\
\hline Leptin $(\mathrm{ng} / \mathrm{ml})$ & $20.02 \pm 11.69$ & $9.38 \pm 4.75$ & $0.000^{*}$ \\
\hline
\end{tabular}

*statistically significant with $\mathrm{p}$ value $<0.05$ 
Table 3: Serum leptin level in male $\&$ female in psoriatic patients (Mean \pm SD)

\begin{tabular}{|l|l|l|l|}
\hline Parameter & Male $(\mathrm{n}=19)$ & Female $(\mathrm{n}=11)$ & P value \\
\hline Leptin $(\mathrm{ng} / \mathrm{ml})$ & $18.40 \pm 11.46$ & $22.46 \pm 12.11$ & 0.360 \\
\hline
\end{tabular}

Table 4: Leptin level and PASI Score (Mean \pm SD)

\begin{tabular}{|l|l|l|l|}
\hline Parameter & PASI $>15$ & PASI $<15$ & P value \\
\hline Leptin(ng/ml) & $32.08 \pm 10.11$ & $13.04 \pm 4.82$ & $0.000^{*}$ \\
\hline
\end{tabular}

*statistically significant with $\mathrm{p}$ value $<0.05$

Table 5: Leptin level and BMI in Psoriasis patients (Mean \pm SD)

\begin{tabular}{|l|l|l|l|}
\hline Parameter & BMI $(<25)$ & BMI $(>25)$ & P value \\
\hline Leptin $(\mathrm{ng} / \mathrm{ml})$ & $18.48 \pm 11.69$ & $20.49 \pm 11.92$ & 0.698 \\
\hline
\end{tabular}

Table 6: Lipid profile in two groups (Mean \pm SD)

\begin{tabular}{|l|c|c|c|}
\hline Parameters & Psoriasis & Healthy volunteers & P value \\
\hline TC $(\mathrm{mg} / \mathrm{dl})$ & $178.07 \pm 22.81$ & $158.23 \pm 25.97$ & $0.003^{*}$ \\
\hline TG $(\mathrm{mg} / \mathrm{dl})$ & $158.17 \pm 24.62$ & $126.77 \pm 19.67$ & $0.000^{*}$ \\
\hline HDL $(\mathrm{mg} / \mathrm{dl})$ & $37.97 \pm 3.31$ & $41.10 \pm 4.12$ & $0.002^{*}$ \\
\hline LDL $(\mathrm{mg} / \mathrm{dl})$ & $108.83 \pm 22.69$ & $92.30 \pm 21.65$ & $0.005^{*}$ \\
\hline VLDL $(\mathrm{mg} / \mathrm{dl})$ & $31.27 \pm 4.94$ & $24.83 \pm 3.95$ & $0.000^{*}$ \\
\hline
\end{tabular}

*statistically significant with $\mathrm{p}$ value $<0.05$

Table 7: Correlation among leptin with lipid profile and BMI

\begin{tabular}{|l|l|l|l|l|l|l|l|l|}
\hline Parameter & & LEPTIN & TC & TG & LDL-C & HDL-C & VLDL-C & BMI \\
\hline Leptin & $\begin{array}{l}\text { Pearson } \\
\text { correlation (r) }\end{array}$ & 1 & 0.154 & $0.398^{* *}$ & 0.150 & $-0.423^{* *}$ & $0.402^{* *}$ & 0.128 \\
\cline { 2 - 8 } & $\begin{array}{l}\text { Sig } \\
(2-\text { tailed })\end{array}$ & & 0.240 & 0.002 & 0.253 & 0.001 & 0.001 & 0.329 \\
\hline
\end{tabular}

**Correlation is significant at the 0.01 level (2-tailed).

Table 8: Correlation between BMI with lipid profile parameter

\begin{tabular}{|l|l|l|l|l|l|l|l|}
\hline parameter & & TC & TG & LDL-C & HDL-C & VLDL-C & BMI \\
\hline BMI $\left(\mathrm{kg} / \mathrm{m}^{2}\right)$ & $\begin{array}{l}\text { Pearson } \\
\text { correlation(r) }\end{array}$ & 0.170 & 0.156 & 0.155 & -0.016 & 0.159 & 1 \\
\cline { 2 - 8 } & sig (2-tailed) & 0.195 & 0.235 & 0.238 & 0.906 & 0.224 & \\
\hline
\end{tabular}




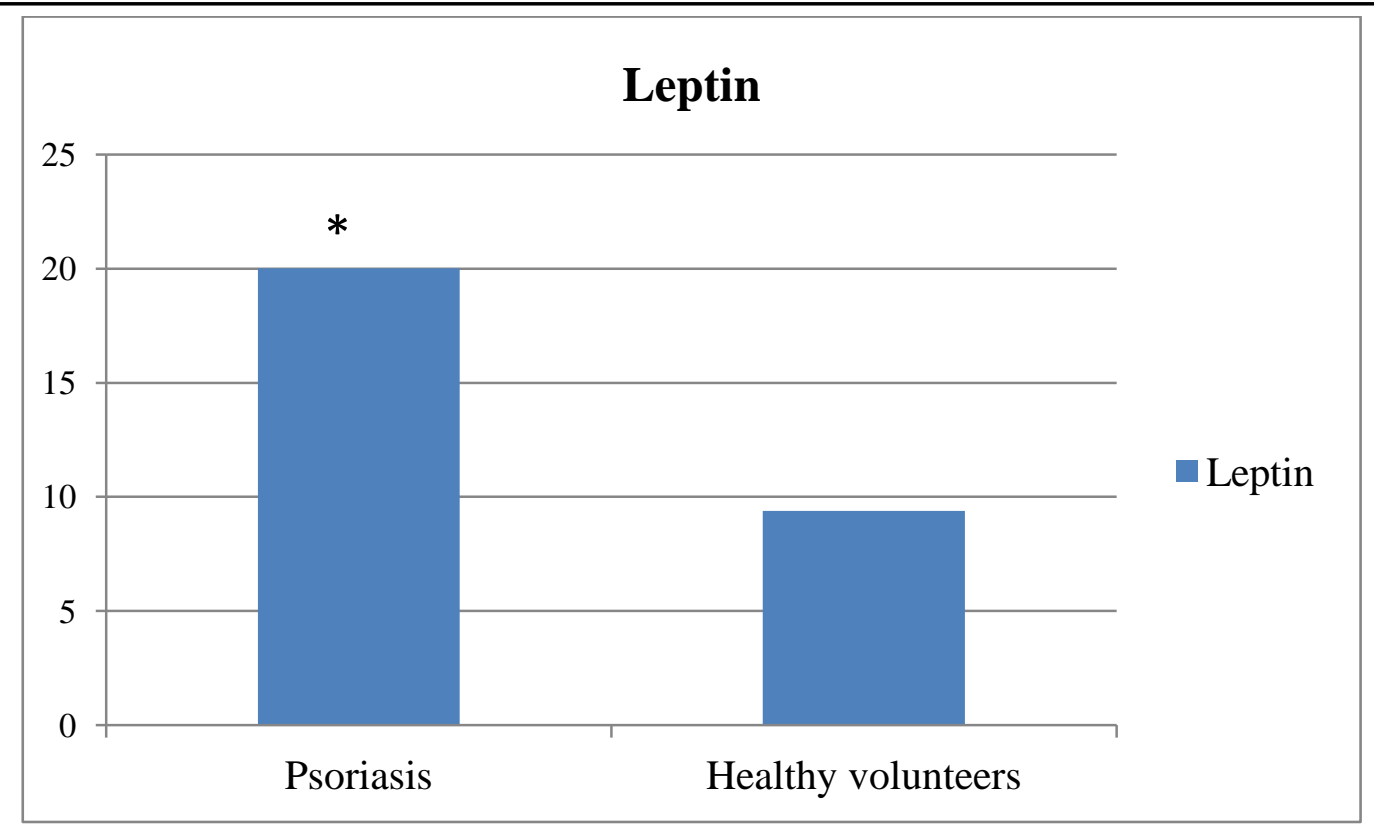

Figure 1: Serum leptin(ng/ml) level in two groups

*Statistically significant with $\mathrm{p}$ value $<0.05$

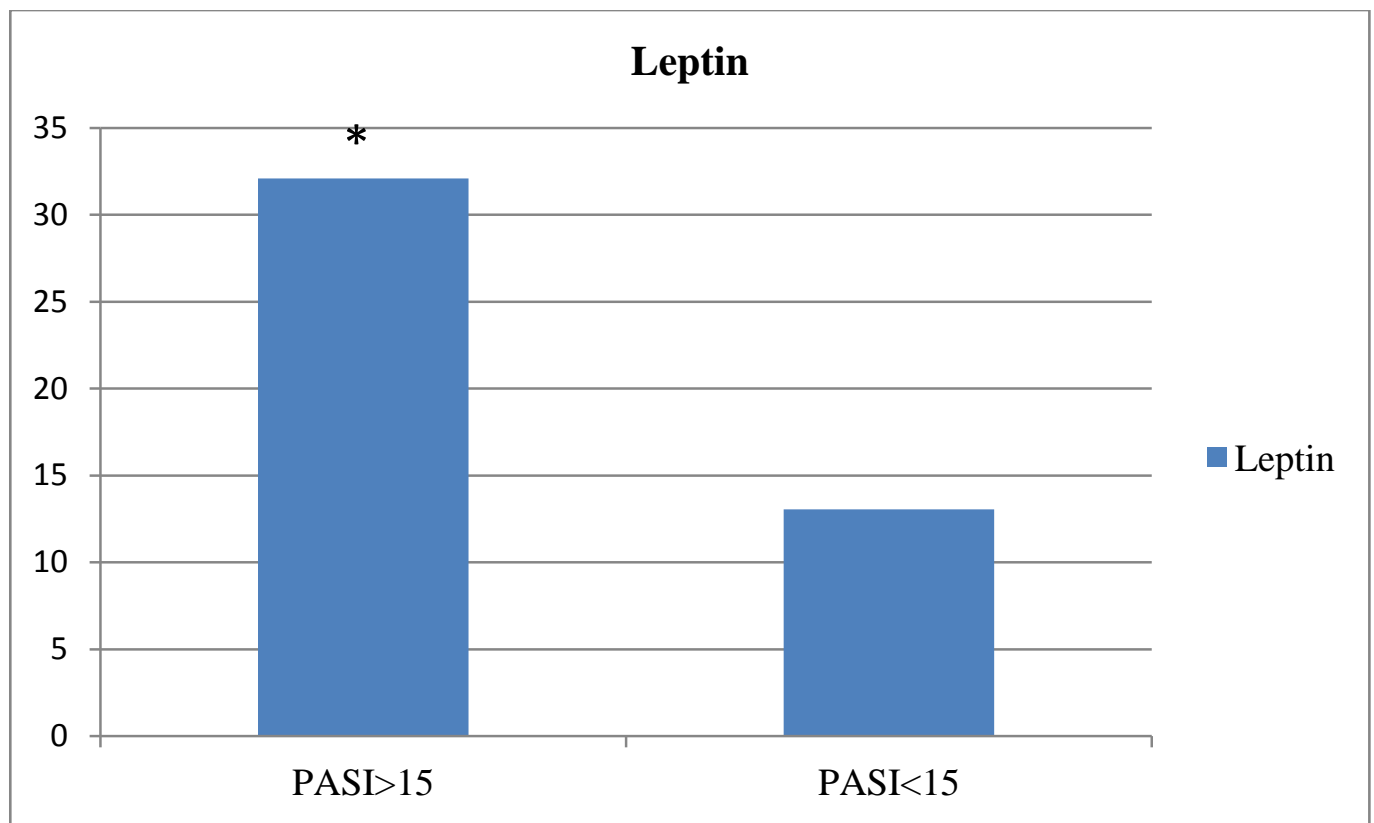

Figure 2: Leptin level (ng/ml) and PASI Score

*Statistically significant with $\mathrm{p}$ value $<0.05$

\section{Discussion}

In this case control study we aimed to see status of serum leptin and lipid profile level in newly diagnosed psoriasis patients and compared it with healthy volunteer control. We found significantly higher serum leptin level in psoriasis patients compared to healthy volunteers (expressed as Mean \pm SD; $20.02 \pm$ 11.69and $9.38 \pm 4.75$ respectively, $\mathrm{p}=0.000$ ). This was in accordance with several studies. ${ }^{[7,20-23]}$ Wang et al found higher serum leptin levels in women and men with psoriasis compared to control group. ${ }^{[7]}$ In Japanese population higher plasma leptin levels have been observed in patients suffering from psoriasis. ${ }^{[20]} \mathrm{In}$ a meta-analysis Zhu KJ et al identified total 11 studies, comprising 773 patients with psoriasis and 570 healthy controls and observed that serum leptin level was increased in patients with psoriasis compared with those in controls. ${ }^{[22]} \mathrm{Y} \mathrm{J}$ Oh et al also found higher serum levels of leptin in 
Korean patients with psoriasis than in controls. ${ }^{[23]}$ On the contrary, Aktanet al did not find any difference in serum leptin level between the psoriatic patients and healthy controls. The results in the study may be affected by small sample size. ${ }^{[24]}$

In psoriatic group, leptin level was significantly higher in patients with higher PASI score $(>15)$ compared to low PASI score $(<15)$ (expressed as Mean \pm SD; $32.08 \pm 10.11$ and $13.04 \pm 4.82$ respectively, $\mathrm{p}=0.000)$. Similar findings were reported by Cerman et al and Zayed et al. ${ }^{[11,12]}$

Serum leptin may contribute to the induction and maintenance of psoriatic lesions, possibly by increasing the production of TNF- $\alpha$ and IL- 6 , the key cytokines in the pathogenesis of psoriasis. Zayed et al reported that there is a strong positive correlation between the leptin levels and TNF- $\alpha$ and IL-6 in psoriasis vulgaris patients. ${ }^{[12]}$

In our study we found higher levels of leptin in psoriatic patients with high BMI but it was statistically insignificant $(\mathrm{p}>0.05)$. Previous studies showed increased circulating leptin levels in patients with high BMI and percentage total body fat and a positive association between BMI and psoriasis. ${ }^{[5,25-26]}$ Lackof association between BMI and psoriasis in our study might because of the fact that BMI in the study population was within normal range. Females had slightly higher leptin level compared to males $(22.46 \pm 12.11$ vs $18.4 \pm 11.46 \mathrm{ng} / \mathrm{ml} ; \mathrm{p}>0.05)$ and this is in confirmation with previous studies. More the body fat content more is the leptin synthesis, since females have more adipose tissue they tend to have elevated levels of this adipocyte derived hormone. ${ }^{[5,7,26-29]}$

Proinflammatory cytokines like TNF- $\alpha$, IL- 6 and IL-17 which are found to be increased in the blood and skin of patients with psoriasis have been implicated in adipose tissue dysfunction and insulin resistance. ${ }^{[15]}$ Insulin resistance is considered as one of the important criteria for defining metabolic syndrome besides hypertension, increased waist circumference and dyslipidemia. It is thought that dyslipidemia is involved in the pathogenesis of psoriasis. There have been many publications demonstrating the role of lipids in psoriasis. ${ }^{[16]}$ In psoriasis, ceramide content and lipid structure of epidermis is altered. TG, TC and phospholipid content of serum and dermis is high in psoriatic patients. ${ }^{[30]}$

Among lipid profile parameters TC, TG, LDL-C and VLDL-C were significantly higher in psoriatic group compared to healthy control $(p<0.05)$. HDL level was higher in healthy control compared to psoriasis $(\mathrm{p}<0.05)$. All lipid profile parameters were statistically comparable between normal and high BMI subgroups in psoriatic patents. Similarly all lipid profile parameters were also statistically comparable between low and high PASI subgroup in psoriatic patents. Leptin had a significant positive correlation with lipid profile parameters such as TG, VLDL-C ( $\mathrm{r}$ value for TG $=0.398$, VLDL-C $=0.402$ ) In addition, leptin had also positive correlation with BMI $(r=0.128)$ and lipid profile parameters like TC and LDL-C ( $\mathrm{r}$ value for $\mathrm{TC}=0.154$, LDL-C $=0.150$ ) while having significant negative correlation with HDL-C ( $\mathrm{r}$ value $=-0.423$ ) in our study population.

Literature shows conflicting results regarding serum lipid profile in psoriatic patients. Some studies indicate normal, some show high and even low serum TG levels in psoriatic patients. ${ }^{[31-36]}$ Commonly reported lipid abnormalities in patients with psoriasis include: elevated LDL-C, TC and TG levelsas well as decreased HDL-C levels. ${ }^{32-}$ ${ }^{34]}$ Piskin et al observed that TC and TG levels were higher in psoriatic patients. ${ }^{[33]}$ In another study, Tekin et al found significantly higher levels of TC in the psoriatic patients $(\mathrm{p}<0.05)$, which are in accordance to our findings. They also reported that oxidized LDL-C is accumulated in psoriatic skin lesions thus associating it to the disease pathogenesis. ${ }^{[35]}$

Reynoso-von DratelnC et al reported that HDL-C was decreased in patients with psoriasis compared with controls as in our study. ${ }^{[37]}$ Ferretti et al reported increase TC and HDL-C in psoriatic children. Therefore, unlike in adults, children did not have cardiovascular risk due to increased TC, 
since HDL-C is protective. The findings of Ferreti et al contradicted our results in regard to HDL-C but this can be explained by the different age distribution in our study population. ${ }^{[38]}$ Mehta et al observed that HDL-C dysfunction and reverse cholesterol transport (RCT) occur in human inflammatory syndromes during endotoxemiaand diabetes. These perturbations in cholesterol efflux capacity from macrophages have a strong inverse association with both carotidintima-media wall thickness (IMT) and the likelihood of angiographic coronary artery disease. ${ }^{[39]}$ Holzer et al described, that HDL-C is a very important factor in RCT. It takes part in the transport of cholesterol produced or accumulated in the peripheral tissues to the liver or other steroidogenic tissues and exerts the antioxidant, anti-inflammatory, antithrombotic and fibrinolytic action. $^{[40]}$

Hence psoriasis, a chronic inflammatory disease is associated with accelerated cardiovascular diseases and could be an independent risk factor for subclinical atherosclerosis. In a study by Enany et al it has been observed that there was significantly increased serum leptin and mean intima-media wall thickness (MIMT) of the common carotid artery (CCA) in patients with psoriasis. MIMT of the CCA was also positively correlated with this biomarker in the study. ${ }^{[21]}$ In another study conducted by Asha et al association between carotid intima thickness and leptin and apolipoprotein-B / apolipoprotein-A revealed a positive correlation among them in psoriasis patients. ${ }^{[41]}$ Kalkan et al also observed a relationship between psoriasis and cardiovascular risk and reported that there was existence of a parallel pathogenic setting underlying the two conditions. ${ }^{[42]}$ Theatherogenic lipid profile we found in psoriatic group of patients in our study is in accordance with the above findings.

We have some limitations in our study. We have small sample size (30 in each group) due to stringent exclusion criteria. Duration of disease could not be correlated with leptin levels since our patients were newly diagnosed having very short history of disease. There may be several unknown confounding factors in our study. The differences in results with various studies might reflect influence of genetic factors, life style, diet and severity of disease in each region.

\section{Conclusion}

This study indicates that hyperleptinemia is associated with psoriasis. Serum leptin level is positively correlated with the disease severity. Thus, it may have role in the pathogenesis or severity of psoriasis. Dyslipidemia is also common in psoriasis. We recommend early monitoring of both leptin and lipid profile in these patients to prevent development of metabolic syndrome and long term cardiovascular events. However further large scale studies on leptin and lipid profile in psoriatic patients are needed to explore the significant association and to develop more effective therapeutic modalities.

\section{Acknowledgements}

Prior publication: None

Supports: None

Conflicts of interest: None

Permissions: Nil

\section{References}

1. Oka A, Mabuchi T, Ozawa A et al. Current understanding of human genetics and genetic analysis of psoriasis. J Dermat 2012;39:231-41.

2. Griffiths CE, Barker JN. Pathogenesis and clinical features of psoriasis. Lancet 2007;370:263-71.

3. Gudjonsson JE, Elder JT. Psoriasis: epidemiology. ClinDermatol 2007; 25(6):535-546.

4. Menter A, Gottlieb A, Feldman SRet al. Guidelines of care for the management of psoriasis and psoriatic arthritis: Section 1. Overview of psoriasis and guidelines of care for the treatment of psoriasis with biologics. J Am AcadDermatol 2008;58(5):826-850. 
5. Hercogová J, Ricceri F, Tripo L et al. Psoriasis and body mass index. DermatolTher 2010;23:152-4.

6. Maffei M, Halaas J, RavussinEet al. Leptin levels in human and rodent: measurement of plasma leptin and ob RNA in obese and weight-reduced subjects. Nat Med 1995; 1:1155-61.

7. Wang Y, Chen J, Zhao Y et al. Psoriasis is associated with increased levels of serum leptin. Br J Dermatol 2008;158:1134-5.

8. Frühbeck G. Intracellular signalling pathways activated by leptin.Biochem $\mathrm{J}$ 2006; 393:7-20.

9. Garofalo C, Surmacz E. Leptin and cancer. J Cell Physiol 2006;207:12-22.

10. Coimbra S, Oliveira $H$, Reis $F$ et al. Circulating adipokine levels in Portuguese patients with psoriasis vulgaris according to body mass index, severity and therapy. $\mathbf{J}$ EurAcadDermatolVenereol 2010;24:138694.

11. Cerman AA, Bozkurt S, SavAet al. Serum leptin levels, skin leptin and leptin receptor expression in psoriasis. $\mathrm{Br} \mathrm{J}$ Dermatol 2008; 159:820-6.

12. Zayed AA, Maksoud NA, Ragab HM. Psoriasis vulgaris severity and body mass index: evaluating therole of leptin, tumor necrosis factor- $\alpha$ and interleukin-6. Journal of the Egyptian Women's Dermatologic Society 2012;9:86-91.

13. Love TJ, Qureshi AA, Karlson EW et al. Prevalence of the metabolic syndrome in psoriasis: results from the National Health and Nutrition Examination Survey, 20032006. Arch Dermatol 2011; 147:419-24.

14. Gelfand JM, Neimann AL, Shin DB et al. Risk of myocardial infarction in patients with psoriasis. JAMA 2006; 296:1735-41.

15. Krueger JG. Hiding under the skin: A welcome surprise in psoriasis. Nat Med 2012; 18:1750-1.

16. Pietrzak A, Michalak-Stoma A, Chodorowska $\mathrm{G}$ et al. Lipid disturbances in psoriasis: an update. Mediators Inflamm 2010;53:6-12.

17. Imagawa K, Matsumoto Y, Numata Yet al. Development of a sensitive ELISA for human leptin, using monoclonal antibodies. ClinChem 2001;47:1579-96.

18. Warnick GR, Nauch M, Rifai N. Evolution of method for measurement of HDLcholesterol: from ultracentrifugation to homogeneous assays. ClinChem 2001; 47:1579-96.

19. Rifai N, Iannotti E, DeAngelis K et al. Analytical and clinical performance of a homogeneous enzymatic LDL-cholesterol assay compared with the ultracentrifugation-dextran sulphate- $\mathrm{Mg}++$ method. ClinChem 1998; 44:1242-50.

20. Takahashi H, Tsuji H, Takahashi I et al. Plasma adiponectin and leptin levels in Japanese patients with psoriasis. $\mathrm{Br} \mathrm{J}$ Dermatol 2008;159:1207-8.

21. Enany B, ElZohiery AK, Elhilaly R et al. Carotid intima-media thickness and serum leptin in psoriasis. Herz 2012;37:527-33.

22. Zhu KJ, Zhang C, Li M et al.Leptin levels in patients with psoriasis: a meta-analysis. ClinExpDermatol 2013;38:478-83.

23. Y J Oh, HK Lim, JH Choi et al. Serum Leptin and Adiponectin Levels in Korean Patients with Psoriasis. J Korean Med Sci 2014; 29: 729-34.

24. Aktan S, Rota S, Erdogan BS et al. A Role OfLeptin in Psoriasis. Turk J Med Sci 2007;37:135-8.

25. Weigle N, McBane S. Psoriasis. Am Fam Physician 2013;87:626-33.

26. Considine RV, Sinha MK, HeimanMLet al. Serum immunoreactive-leptin concentrations in normal-weight and obese humans. New Engl J Med 1996;334:292-5.

27. Müller G, Ertl J, GerlM et al. Leptin impairs metabolic actions of insulin in isolated rat adipocytes. $\mathrm{J}$ BiolChem 1997;272:10585-93. 
28. Zhao AZ, Shinohara MM, Huang Det al. Leptin induces insulin-like signaling that antagonizes cAMP elevation by glucagon in hepatocytes. $\mathrm{J}$ BiolChem 2000;275:11348-54.

29. Frühbeck G. Intracellular signalling pathways activated by leptin.Biochem $\mathrm{J}$ 2006; 393:7-20.

30. Motta S, Monti M, Sesana S et al.Abnormality of water barrier function in psoriasis. Role of ceramidefractions. Arch Dermatol 1994;130:452-6.

31. Pietrzak A, Chodorowska G, Szepietowski $\mathrm{J}$ et al. Psoriasis and serum lipid abnormalities. Dermatol Ther 2010;23:160-73.

32. Mallbris L, Granath F, Hamsten A et al. Psoriasis is associated with lipid abnormalities at the onset of skin disease. $\mathrm{J}$ Am AcadDermatol 2006;54:614-21.

33. Piskin S, Gurkok F, Ekuklu G et al. Serum lipid levels in psoriasis. Yonsei Med J 2003;44:24-6.

34. VanizorKural B, Orem A, Cimsit GU et al. Evaluation of the atherogenic tendency of lipids and lipoprotein content and their relationships with oxidant-antioxidant system in patients with psoriasis. ClinicaChemicaActa2003;328:71-82.

35. Tekin NS, Tekin IO, Barut F et al. Accumulation of oxidized low-density lipoprotein in psoriatic skin and changes of plasma lipid levels in psoriatic patients. Mediators Inflamm 2007;2007:78454.

36. Rocha-Pereira P, Santos-Silva A, Rebelo I et al. Dislipidemia and oxidative stress in mild and in severe psoriasis as a risk for cardiovascular disease. Clinica Chimica Acta 2001;303:33-39.

37. Reynoso-von Drateln C, MartínezAbundis E, Balcázar-Muñoz BR et al. Lipid profile, insulin secretion, and insulin sensitivity in psoriasis. J Am Acad Dermatol 2003;48:882-5.
38. Ferretti G, Simonetti O, Offidani AM et al. Changes of plasma lipids and erythrocyte membrane fluidity in psoriatic children. Pediatr Res 1993;33:506-9.

39. Mehta NN, Li R, Krishnamoorthy $P$ et al. Abnormal lipoprotein particles and cholesterol efflux capacity in patients with psoriasis. Atherosclerosis 2012;224:21821.

40. Holzer M, Wolf $\mathrm{P}$, Curcic $\mathrm{S}$ et al. Psoriasis alters HDL composition and cholesterol efflux capacity. J Lipid Res 2012;53:161824.

41. Kumari A, Suman B. Sharma, SingalA et al. Association of carotid intima-media thickness with leptin and apolipoprotein b/apolipoprotein a-i ratio reveals imminent predictors of subclinicalatherosclerosis in psoriasis patients. Actamedica 2014; 57:21-7.

42. Kalkan G, Karadag S. Cardiovascular risk in psoriasis. Eur J Gen Med 2013;10:10-6. 